\title{
A Class of Explicit Perfect Multi-sequences
}

\author{
Chaoping Xing ${ }^{1}$, Kwok Yan $\mathrm{Lam}^{2}$, and Zhenghong $\mathrm{Wei}^{3}$ \\ 1 Department of Mathematics, National University of Singapore \\ 2 Science Drive 2, 117543, Singapore \\ xingcp@math.nus.edu.sg \\ 2 School of Computing, National University of Singapore \\ 2 Science Drive 2, 117543, Singapore \\ lamky@comp.nus.edu.sg \\ 3 Department of Mathematics, Shenzhen Normal College, People's Republic of China \\ weizhenghong@163.net
}

\begin{abstract}
In [7], perfect multi-sequences are introduced and a construction based on function fields over finite fields is given. In this paper, we explore the construction in [7] by considering rational function fields. Consequently a class of perfect multi-sequences are obtained.
\end{abstract}

\section{Introduction}

There are a lot of papers concerning linear complexity of one single sequence [2], [3], [4], [5], [8]. For multi-sequences, it seems that few results are known. One of these results is the Massey's algorithm for linear feedback shift register (LFSR) synthesis that was proven by Ding [1]. Recently perfect multi-sequences are introduced in [7]. This definition is a natural generalization of one single perfect sequence defined by Rueppel [4], [5].

We adopt notations from [7].

Definition 1. Let $\mathbf{F}_{q}$ be the finite field with $q$ elements. The linear complexity of a sequence $\mathbf{c}=\left(c_{1}, c_{2}, \ldots, c_{n}\right)$ of elements of $\mathbf{F}_{q}$ is defined to be the least $k$ such that it is a $k$-th order LFSR sequence.

We now consider multi-sequences $\mathbf{c}_{1}, \mathbf{c}_{2}, \ldots, \mathbf{c}_{m}$ of dimension $m$ of length $n$. $C$ denotes the multi-sequences $\left\{\mathbf{c}_{i}\right\}_{i=1}^{m}$ of length $n$.

Definition 2. The linear complexity of a multi-sequence set $C=\left\{\mathbf{c}_{i}=\left(c_{i 1}, c_{i 2}\right.\right.$, $\left.\left.\ldots, c_{i n}\right)\right\}_{i=1}^{m}$ of length $n$ is defined to be the smallest order $k$ of $\operatorname{LFSR}(f(T), k)$ generating all of sequences $\mathbf{c}_{i}$ for $i=1,2, \ldots, m$.

The Massey's algorithm gives a way to find the shortest LFSR generating multi-seuqences, and therefore the linear complexity of a multi-sequence set $C$ is obtained.

Now we turn to sequences of infinite length.

Consider multi-sequences of dimension $m \geq 1$, 


$$
\begin{gathered}
\mathbf{a}_{1}=\left(a_{11}, a_{12}, a_{13}, \ldots\right) \in \mathbf{F}_{q}^{\infty} \\
\mathbf{a}_{2}=\left(a_{21}, a_{22}, a_{23}, \ldots\right) \in \mathbf{F}_{q}^{\infty} \\
\cdot \\
\cdot \\
\mathbf{a}_{m}=\left(a_{m 1}, a_{m 2}, a_{m 3}, \ldots\right) \in \mathbf{F}_{q}^{\infty}
\end{gathered}
$$

and let $A$ be the multi-sequence set $\left\{\mathbf{a}_{i}\right\}_{i=1}^{m}$. We also denote by $A_{n}$ the multisequences

$$
\left\{\left(a_{i 1}, a_{i 2}, \ldots, a_{i n}\right)\right\}_{i=1}^{m}
$$

of length $n$ and dimension $m$.

Definition 3. The linear complexity profile of a multi-sequence set $A=$ $\left\{\mathbf{a}_{1}, \mathbf{a}_{2}, \ldots \mathbf{a}_{m}\right\}$ is defined by the sequence of integral numbers

$$
\left\{\ell_{n}(A)\right\}_{n=1}^{\infty}
$$

where $\ell_{n}(A)$ denote the linear complexities of $A_{n}=\left\{\left(a_{i 1}, a_{i 2}, \ldots, a_{i n}\right)\right\}_{i=1}^{m}$ of length $n$.

In [7], perfect multi-sequences are defined in the following way.

Definition 4. The multi-sequence set $A=\left\{\mathbf{a}_{1}, \mathbf{a}_{2}, \ldots \mathbf{a}_{m}\right\}$ is called perfect if

$$
\ell_{n}(A)=\left\lceil\frac{m n}{m+1}\right\rceil
$$

for all $n \geq 1$, where $\lceil v\rceil$ denotes the smallest integer bigger than or equal to the real number $v$.

Furthermore, it is proven in [7] that

Theorem 1. The multi-sequence set $A=\left\{\mathbf{a}_{1}, \mathbf{a}_{2}, \ldots \mathbf{a}_{m}\right\}$ is perfect if and only if

$$
\ell_{n}(A) \geq\left\lceil\frac{m n}{m+1}\right\rceil
$$

for all $n \geq 1$.

This paper is organized as follows. In Section 2, we recall the main result of [7] concerning constructions of perfect multi-sequences. In Section 3, a class of perfect multi-sequences are given based on the result of Section 2. Some explicit examples are illustrated in this section. 


\section{Construction of Perfect Multi-sequences}

First we introduce some notations (see [6]).

For a divisor $D=\sum_{P} m_{P} P$, the degree of $D$ is defined by $\operatorname{deg}(D)=$ $\sum_{P} m_{P} \operatorname{deg}(P)$.

Let $z$ be a non-zero element of $F$, define the zero divisor of $z$ by

$$
(z)_{0}=\sum_{\nu_{P}(z)>0} \nu_{P}(z) P
$$

and the pole divisor of $z$ by

$$
(z)_{\infty}=-\sum_{\nu_{P}(z)<0} \nu_{P}(z) P .
$$

Then $(z)_{0}$ and $(z)_{\infty}$ are two effective divisors and $\operatorname{deg}(z)_{0}=\operatorname{deg}(z)_{\infty}$. The principal divisor of $z$ is defined by

$$
\operatorname{div}(z)=(z)_{0}-(z)_{\infty} .
$$

For a divisor $D$, the linear space $L(D)$ is defined by

$$
L(D)=\{f \in F \mid \operatorname{div}(f)+D \geq 0\} .
$$

It is an $\mathbf{F}_{q}$-linear space of finite dimension. For two divisors $D=\sum_{P} m_{P} P$ and $G=\sum_{P} n_{P} P$, we denote by $D \vee G$ the divisor $\sum_{P} \max \left\{m_{P}, n_{P}\right\} P$.

For a place $Q$ of degree $m$ of $F$, the integral ring of $Q$ is

$$
\mathcal{O}_{Q}=\left\{z \in F \mid \nu_{Q}(z) \geq 0\right\} .
$$

This is a local ring with the maximal ideal

$$
\wp_{Q}=\left\{z \in \mathcal{O}_{Q} \mid \nu_{Q}(z)>0\right\} .
$$

The residue class field $\mathcal{O}_{Q} / \wp_{Q}$ is an extension over $\mathbf{F}_{q}$ of degree $m=\operatorname{deg}(Q)$. For an element of $z \in \mathcal{O}_{Q}$, the residue class $\bar{z}$ of $z$ in $\mathcal{O}_{Q} / \wp_{Q}$ is denoted by $z(Q)$.

From now on in this section, we have the following notations and assumptions.

$F / \mathbf{F}_{q}$ - a global function field with the full constant field $\mathbf{F}_{q}$;

$Q$ - a place of degree $m$ of $F$;

$x_{1}, x_{2}, \ldots, x_{m}-m$ elements of $\mathcal{O}_{Q}$ satisfying that $x_{1}(Q), x_{2}(Q), \ldots, x_{m}(Q)$

form an $\mathbf{F}_{q}$-basis of $\mathcal{O}_{Q} / \wp_{Q}$;

$t$ - a local parameter of $Q$ with $\operatorname{deg}(t)_{\infty}=m+1$;

$y$ - an element of $\mathcal{O}_{Q}$ satisfying $y \notin \bigoplus_{i=1}^{m} \mathbf{F}_{q}(t) x_{i}$.

Then $y$ has a local expansion at $Q$ with the following form,

$$
y=\sum_{j=0}^{\infty}\left(\sum_{i=1}^{m} a_{i j} x_{i}\right) t^{j},
$$


where $a_{i, j} \in \mathbf{F}_{q}$.

Denote

$$
\mathbf{a}_{i}(y)=\left(a_{i 1}, a_{12}, a_{i 3}, \ldots\right) \in \mathbf{F}_{q}^{\infty}
$$

for any $1 \leq i \leq m$. We form a multi-sequence set

$$
A(y)=\left\{\mathbf{a}_{i}(y)\right\}_{i=1}^{m} .
$$

It is proved in [7] that $A(y)$ is a perfect multi-sequence set if $m=\operatorname{deg}\left((y)_{\infty} \vee\right.$ $\left.\left(x_{1}\right)_{\infty} \vee\left(x_{2}\right)_{\infty} \vee \cdots \vee\left(x_{m}\right)_{\infty}\right)$.

We organize the above result as follows.

Theorem 2. Let $G$ be a positive divisor of degree $m$ with $Q \notin \operatorname{Supp}(G)$. Suppose that $x_{1}, x_{2}, \ldots, x_{m}, y$ are elements of $L(G)$ such that $x_{1}(Q), x_{2}(Q), \ldots, x_{k}(Q)$ form an $\mathbf{F}_{q}$-basis of $\mathcal{O}_{Q} / \wp_{Q}$ and $y \notin \bigoplus_{i=1}^{m} \mathbf{F}_{q}(t) x_{i}$. Then $A(y)=\left\{\mathbf{a}_{i}(y)\right\}_{i=1}^{m}$ constructed as (1) is perfect.

\section{Explicit Perfect Multi-sequences}

In this section, we present a class of perfect multi-sequences based on the results of Section 2. The function fields used are the rational function fields.

Throughout this section, $F$ denotes the rational function field $\mathbf{F}_{q}(x)$. We also fix a place $Q$ of degree $m$ and a rational place $P$ of $F$ with $Q \neq P$. Let $t \in F$ be a function with $(t)_{0}=Q+P$.

Proposition 1. Let $G$ be a positive divisor of degree $m$ such that $\operatorname{Supp}(G) \cap$ $\{Q, P\}=\emptyset$. Let $\left\{x_{1}=1, x_{2}, \ldots, x_{m}, y\right\}$ be a basis of $L(G)$. Then $\left\{x_{1}=1, x_{2}, \ldots\right.$, $\left.x_{m}, y\right\}$ is also a basis of $F$ over $\mathbf{F}_{q}(t)$,

Proof. Since the degree $\left[F: \mathbf{F}_{q}(t)\right]$ of the extension $F / \mathbf{F}_{q}(t)$ is equal to the degree of the zero divisor of $t$, we have $\left[F: \mathbf{F}_{q}(t)\right]=\operatorname{deg}(t)_{0}=\operatorname{deg}(Q+P)=$ $m+1$. This means that $F$ is a vector space of dimension $m+1$ over $\mathbf{F}_{q}(t)$. Hence it is sufficient to prove that $x_{1}=1, x_{2}, \ldots, x_{m}, y$ are linearly independent over $\mathbf{F}_{q}(t)$. Suppose that there exist $m+1$ functions $u_{1}(t), \ldots, u_{m}(t), u(t) \in \mathbf{F}_{q}(t)$ such that not all of them equal to zero and

$$
u(t) y+\sum_{i=1}^{m} u_{i}(t) x_{i}=0 .
$$

By multipling a polynomial in $\mathbf{F}_{q}[t]$, we may assume that all $u_{i}(t)$ and $u(t)$ are polynomials in $\mathbf{F}_{q}[t]$. Furthermore, dividing $u(t)$ and all $u_{i}(t)$ by the greatest common divisor $\left(u(t), u_{1}(t), \ldots, u_{m}(t)\right)$ of $u(t), u_{1}(t), \ldots, u_{m}(t)$, we can assume that $\left(u(t), u_{1}(t), \ldots, u_{m}(t)\right)=1$. Thus not all of $u(0), u_{1}(0), \ldots, u_{m}(0)$ are equal to zero. As $x_{1}=1, x_{2}, \ldots, x_{m}, y$ are a basis of $L(G)$, we have $u(0) y+$ $\sum_{i+0}^{m} u_{i}(0) x_{i} \neq 0$. 
Rewrite (2) into the form

$$
(u(t)-u(0)) y+\sum_{i=1}^{m}\left(u_{i}(t)-u_{i}(0)\right) x_{i}=-\left(u(0) y+\sum_{i=1}^{m} u_{i}(0) x_{i}\right) .
$$

Look at the left side of (3), we find that $Q+P=(t)_{0}$ is less than or equal to the zero divisor of the function $u(0) y+\sum_{i=1}^{m} u_{i}(0) x_{i}$. Hence $u(0) y+\sum_{i=1}^{m} u_{i}(0) x_{i}$ is an nonzero element of $L(G-(Q+P))$. However $L(G-(Q+P))=\{0\}$ since $\operatorname{deg}(G-(Q+P))=-1$. This contradiction shows that $x_{1}=1, x_{2}, \ldots, x_{m}, y$ are linearly independent over $\mathbf{F}_{q}(t)$.

Theorem 3. Let the divisor $G$ and functions $x_{1}, x_{2}, \ldots, x_{m}, y$ be as in Proposition 1. If there is a positive divisor $G_{1}$ with $\operatorname{deg}\left(G_{1}\right)=m-1$ such that $G_{1} \leq G$ and $x_{1}, x_{2}, \ldots, x_{m}$ form a basis of $L\left(G_{1}\right)$, then the multi-sequence set $A(y)$ constructed as (1) is perfect.

Proof. Since $\left\{x_{1}=1, x_{2}, \ldots, x_{m}, y\right\}$ is a basis of $F$ over $\mathbf{F}_{q}(t)$ by Proposition 1 , we have $y \notin \bigoplus_{i=1}^{m} \mathbf{F}_{q}(t) x_{i}$.

Suppose that $\alpha_{1}, \alpha_{2}, \ldots, \alpha_{m} \in \mathbf{F}_{q}$ satisfy

$$
\sum_{i=1}^{m} \alpha_{i} x_{i}(Q)=0
$$

Then $\sum_{i=1}^{m} \alpha_{i} x_{i}$ is an element of $L\left(G_{1}-Q\right)$. Thus $\sum_{i=1}^{m} \alpha_{i} x_{i}$ is equal to 0 since $\operatorname{deg}\left(G_{1}-Q\right)=-1$. This implies that $x_{1}(Q), x_{2}(Q), \ldots, x_{m}(Q)$ form an $\mathbf{F}_{q}$-basis of $\mathcal{O}_{Q} / \wp_{Q}$.

Appling Theorem 2, we have that $A(y)$ is perfect as $\operatorname{deg}(G)=m$.

Example 1. Let $Q(x)$ be an irreducible polynomials of degree $m$ in $\mathbf{F}_{q}[x]$ and $\alpha$ an element of $\mathbf{F}_{q}$ satifying $Q(\alpha) \neq 0$. Put

(a) $t=Q(x)(x-\alpha)$;

(b) $x_{i}=x^{i-1}$ for all $1 \leq i \leq m$;

(c) $y=x^{m}$.

Then it follows from Theorem 3 that $A(y)$ is a perfect multi-sequence set of dimension $m$ since $\left\{x_{1}, x_{2}, \ldots, x_{m}\right\}$ is a basis of $L((m-1) \infty)$ and $\left\{x_{1}, x_{2}, \ldots, x_{m}, y\right\}$ is a basis of $L(m \infty)$, where $\infty$ is the pole of $x$.

(a) binary perfect multi-sequences of dimension $m=3$.

Taking $Q(x)=x^{3}+x+1 \in \mathbf{F}_{2}[x], t=x Q(x)$, and $x_{1}=1, x_{2}=x, x_{3}=$ $x^{2}, y=x^{3}$, we obtain the local expansion of $y$ at $Q$ :

$y=\left(x_{1}+x_{2}\right)+\left(x_{1}+x_{3}\right) t+\left(x_{1}+x_{2}+x_{3}\right) t^{2}+x_{1} t^{3}+\left(x_{1}+x_{2}\right) t^{4}+x_{1} t^{5}+x_{1} t^{6}+\left(x_{1}+x_{3}\right) t^{8}+\cdots$.

Put

$$
\begin{aligned}
& \mathbf{a}_{1}(y)=(1,1,1,1,1,1,0,1, \cdots) \\
& \mathbf{a}_{2}(y)=(0,1,0,1,0,0,0,0, \cdots), \\
& \mathbf{a}_{3}(y)=(1,1,0,0,0,0,0,1, \cdots) .
\end{aligned}
$$


By Theorem 3, $\mathbf{a}_{1}(y), \mathbf{a}_{2}(y), \mathbf{a}_{3}(y)$ are perfect multi-sequences of dimension 3.

(b) ternary perfect multi-sequences of dimension $m=2$.

Taking $Q(x)=x^{2}+1, t=x\left(x^{2}+1\right)$, and $x_{1}=1, x_{2}=2, y=x^{2}$, we get the local expansion of $y$ at $Q$ :

$$
y=2 x_{1}+2 x_{2} t+2 x_{1} t^{2}+x_{2} t^{3}+2 x_{1} t^{4}+2 x_{1} t^{6}+2 x_{2} t^{9}+\cdots .
$$

Put

$$
\begin{gathered}
\mathbf{a}_{1}(y)=(0,2,0,2,0,2,0,0, \cdots), \\
\mathbf{a}_{2}(y)=(2,0,1,0,0,0,0,0, \cdots) .
\end{gathered}
$$

By Theorem 3, $\mathbf{a}_{1}(y), \mathbf{a}_{2}(y)$ are perfect multi-sequences of dimension 2.

Example 2. Let $Q(x)$ be an irreducible polynomials of degree $m$ in $\mathbf{F}_{q}[x]$ and $\alpha$ an element of $\mathbf{F}_{q}$ satifying $Q(\alpha) \neq 0$. Put

(a) $t=Q(x) / x^{m+1}$;

(b) $x_{i}=(x-\alpha)^{1-i}$ for all $1 \leq i \leq m$;

(c) $y=(x-\alpha)^{-m}$.

Then it follows from Theorem 3 that $A(y)$ is a perfect multi-sequence set of dimension $m$ since $\left\{x_{1}, x_{2}, \ldots, x_{m}\right\}$ is a basis of $L((m-1) P)$ and $\left\{x_{1}, x_{2}, \ldots, x_{m}, y\right\}$ is a basis of $L(m P)$, where $P$ is the zero of $x-\alpha$.

(a) binary perfect multi-sequences of dimension $m=2$.

Taking $Q(x)=x^{2}+x+1, t=\left(x^{2}+x+1\right) / x^{3}$, and $x_{1}=1, x_{2}=1 / x, y=1 / x^{2}$, we obtain the local expansion of $y$ at $Q$ :

$$
y=\left(x_{1}+x_{2}\right)+\left(x_{1}+x_{2}\right) t+x_{2} t^{2}+\left(x_{1}+x_{2}\right) t^{3}+t^{4}+t^{6}+\cdots .
$$

Put

$$
\begin{gathered}
\mathbf{a}_{1}(y)=(1,0,1,1,0,1, \cdots), \\
\mathbf{a}_{2}(y)=(1,1,1,0,0,0, \cdots) .
\end{gathered}
$$

By Theorem $3, \mathbf{a}_{1}(y), \mathbf{a}_{2}(y)$ are perfect multi-sequences of dimension 2 .

(b) ternary perfect multi-sequences of dimension $m=2$.

Taking $Q(x)=x^{2}+2 x+2, t=\left(x^{2}+2 x+2\right) / x^{3}$, and $x_{1}=1, x_{2}=1 / x, y=$ $1 / x^{2}$, we obtain the local expansion of $y$ at $Q$ :

$$
y=\left(x_{1}+2 x_{2}\right)+\left(2 x_{1}+2 x_{2}\right) t+\left(x_{1}+2 x_{2}\right) t^{2}+x_{1} t^{3}+\left(x_{1}+2 x_{2}\right) t^{4}+\cdots .
$$

Put

$$
\begin{gathered}
\mathbf{a}_{1}(y)=(2,1,1,1, \cdots), \\
\mathbf{a}_{2}(y)=(2,2,0,2, \cdots) .
\end{gathered}
$$

By Theorem 3, $\mathbf{a}_{1}(y), \mathbf{a}_{2}(y)$ are perfect multi-sequences of dimension 2. 


\section{References}

1. C. S. Ding, "Proof of Massey's conjectured algorithm," in Advances in Cryptology - Eurocrypt'88, C. G. Guenther ed., Springer-Verlag, Berlin, LNCS Vol. 310, 1988, pp.345-349. 299

2. D. R. Kohel, S. Ling and C. P. Xing, "Explicit sequence expansions," to appear in DMTC, Springer-Verlag. 299

3. H. Niederreiter, "Sequences with almsot perfect linear complexity profile," in $A d$ vances in Cryptology-EUROCRYPT'87, D. Chaumand and W. L. Price, eds., Springer-Verlag, Berlin: LNCS, Vol. 304, 1988, pp. 37-51. 299

4. R. A. Rueppel, Analysis and Design of Stream Ciphers, Berlin: Springer-Verlag, 1986. 299

5. R. A. Rueppel, "Stream ciphers," in Contemporary Cryptology-The Science of Information Integrity, G. J. Simmons, Ed., IEEE Press, New York: 1992, pp. 65-134. 299

6. H. Stichtenoth, Algebraic Function Fields and Codes, Berlin: Springer-Verlag, 1993. 301

7. C. P. Xing, "Perfect multi-sequences and function fields over finite fields", Preprint, 1999. 299, 300, 302

8. C. P. Xing and K. Y. Lam, "Sequences with almost perfect linear complexity profiles and curves over finite fields," IEEE Trans. Inform. Theory, vol. IT-45, 1999, pp.1267-1270. 299 